\title{
Die Gesundheitsreform und der Wettbewerb: Viel Rhetorik, wenig Substanz
}

Mit der Gesundheitsreform 2006 steht nun - knapp drei Jahre nach dem „Gesetz zur Modernisierung der Gesetzlichen Krankenversicherung" (GMG) - schon wieder die nächste große Gesundheitsreform an. Das inzwischen als Fraktionsentwurf vorliegende "Gesetz zur Stärkung des Wettbewerbs in der Gesetzlichen Krankenversicherung" (GKV-WSG) tritt dabei mit dem primären Ziel an, die Wettbewerbsorientierung des Gesundheitssystems stärken zu wollen. Bei genauerer Betrachtung der zentralen finanzierungs- und leistungsseitigen Reformmaßnahmen zeigt sich jedoch, dass die tatsächliche Substanz für Wettbewerbsstärkung weit hinter den im Gesetzentwurf formulierten Ansprüchen zurückbleibt.

\section{Klaus Jacobs und Sabine Schulze}

\section{Die Reformdebatte und das Wettbewerbsstärkungsgesetz}

„Mehr Wettbewerb“ ist unbestritten das am stärksten proklamierte Ziel der Regierungskoalition für die anstehende Gesundheitsreform 2006. Schon im Koalitionsvertrag vom 11. November 2005 hieß es ambitioniert, dass „der Bereich der Gesundheitsversorgung (...) durch die Schaffung flexiblerer Rahmenbedingungen konsequent wettbewerblich ausgestaltet werden (soll)" (CDU/ CSU/SPD 2005, S. 103). Im Eckpunktepapier der Großen Koalition vom Juli 2006 wurde diese Zielsetzung noch einmal bestärkt - hier hieß es vollmundig, dass „die Erweiterung der Wahl- und Entscheidungsmöglichkeiten der Versicherten, die Intensivierung des Wettbewerbs um Qualität und Wirtschaftlichkeit sowohl zwischen Krankenkassen als auch zwischen Leistungserbringern, die Erhöhung der Transparenz über Angebote, Leistungen und Abrechnungen, die Verminderung des bürokratischen Aufwands (...) noch stringenter verfolgt werden (müssen)." Sogar der auf fast 600 Seiten aufgeblähte Gesetzentwurf erhielt einen entsprechenden Titel: Die Reform soll als GKV-Wettbewerbsstärkungsgesetz (GKV-WSG) in die Geschichte eingehen und dafür sorgen, dass „das Gesundheitssystem auf allen Ebenen neu strukturiert und wettbewerblicher ausgerichtet" wird (Begründung Gesetzentwurf BT 16/3100', Allgemeiner Teil, Punkt I.3.).

Wie sieht es nun mit der Wettbewerbsorientierung der Gesundheitsreform 2006 tatsächlich aus? Wird diese Reform ihrem hohen Anspruch gerecht? Welche Folgen

Dr. Klaus Jacobs, Geschäftsführer des wissenschaftlichen Instituts der AOK (WIdO), Sabine Schulze, wissenschaftliche Mitarbeiterin im WIdO. sind durch die geplanten Neuregelungen auf der Finanzierungs- und auf der Leistungsseite der GKV für den Wettbewerb im Gesundheitssystem zu erwarten? Auch wenn die außerordentliche Vielzahl an Neuregelungen dazu zwingt, jeden Anspruch auf eine vollständige Beurteilung fallen zu lassen, soll im Folgenden gleichwohl der Versuch unternommen werden, die zentralen finanzierungs- und leistungsseitigen Neuregelungen einer Bewertung im Hinblick auf ihre Wettbewerbsorientierung zu unterziehen. Im Fokus stehen dabei vor allem die leistungsseitigen Strukturreformen des geplanten GKV-WSG, auf die vielfach verwiesen wird, wenn es heißt, dass der Gesetzentwurf durchaus auch positive Elemente aufweise.

\section{Die Finanzierungsseite: Zentraler Gesundheitsfonds und kassenspezifische Zusatzbeiträge}

Zunächst aber zur Finanzierungsseite. Nach dem GKVModernisierungsgesetz (GMG) von 2003 bestand allgemein die Erwartung, dass sich die nächste Gesundheitsreform vor allem den im GMG weitgehend ausgesparten, aber gleichwohl dringlichen Finanzierungsproblemen der GKV widmen würde. Wofür waren schließlich die Rürupund die Herzog-Kommission eingerichtet worden? Im Bundestagswahlkampf 2005 besetzten die Parteien explizit mögliche neue Finanzierungsmodelle der GKV als prominente Wahlkampfthemen, und noch im Koalitionsvertrag wird beim Themenbereich Krankenversicherung an erster Stelle das Ziel der „Sicherung einer nachhaltigen und gerechten Finanzierung" genannt (CDU, CSU, SPD 2005, S. 102). 


\subsection{Veränderte Problemdiagnose}

Davon ist jedoch seit den Anfang Juli 2006 vereinbarten „Eckpunkten“ keine Rede mehr. Das Hauptproblem der GKV lag plötzlich nicht mehr in der Wachstumsschwäche der beitragspflichtigen Einnahmen - dem man folgerichtig mit einem „Finanzkraftstärkungsgesetz" hätte beikommen müssen, um die GKV-Finanzierung stabiler, nachhaltiger und gerechter zu gestalten. Nunmehr lag das zentrale Problem der GKV im unzureichendem Wettbewerb um Qualität und Wirtschaftlichkeit der Versorgung, weshalb als primäres Reformziel ausgegeben wurde, Transparenz und Intensität des Kassenwettbewerbs zu erhöhen.

Grund genug, sich das neue Finanzierungsmodell aus Gesundheitsfonds und kassenspezifischen Zusatzbeiträgen im Hinblick auf seine Implikationen für den Kassenwettbewerb anzuschauen. Eine Analyse des Fondsmodells bezüglich der „ursprünglichen“ Ziele der Stabilisierung und nachhaltigen Sicherung der GKV-Finanzierung bei erhöhter Verteilungsgerechtigkeit kann hier unterbleiben, denn hierzu leistet die Reform keinen Beitrag. Nicht einmal eine kurzfristige Beitragssatzstabilisierung gelingt, weil die aktuell gewährten Steuerzuschüsse zunächst sogar von 4,2 Mrd. auf 1,5 Mrd. gesenkt werden. Wird denn der Wettbewerb der Kassen um Versicherte intensiviert? Und wie werden sich die Akteure im Wettbewerb auf dem Versicherungsmarkt künftig verhalten - die Kassenmitglieder bei der Kassenwahl und die Krankenkassen im Wettbewerb um die Mitglieder?

Dabei ist zunächst anzumerken, dass im Verhältnis der Krankenkassen untereinander bereits heute intensiver (Beitragssatz)-Wettbewerb herrscht. Warum hätten sich in der Vergangenheit wohl sonst so viele Kassen entgegen der bestehenden Rechtslage verschuldet, anstatt ihre Beitragssätze anzuheben? Was im Hinblick auf den Kassenwettbewerb bisher als defizitär galt, war weniger seine Intensität als vielmehr seine inhaltliche Ausrichtung aufgrund falsch gesetzter Handlungsanreize wegen eines unzureichend morbiditätsorientierten $\mathrm{RSA}^{2}$ sowie ungenügender leistungsseitiger Handlungsparameter der Kassen ${ }^{3}$.

Das Finanzierungsmodell des Gesundheitsfonds mit Zusatzbeiträgen sieht vor, dass der zentrale Gesundheitsfonds künftig den Hauptteil der Finanzierung der Krankenkassen übernehmen soll. Mitglieder aller Kassen und ihre Arbeitgeber (bzw. die Rentenversicherungsträger) zahlen Beiträge auf der Grundlage eines bundeseinheitlichen Beitragssatzes, der künftig per Rechtsverordnung festgelegt wird. Zudem soll der Fonds aus Steuermitteln durch wachsende Bundeszuschüsse gespeist werden aber über die für 2009 zugesagten 3,0 Mrd. Euro hinaus bleibt dies vorerst lediglich eine vage Absichtserklärung.

\subsection{Untauglicher Wettbewerbsparameter Zusatzbeitrag}

Der Beitragswettbewerb um Versicherte soll künftig über kassenspezifische Zusatzbeiträge ausgetragen werden, die die Kassen prozentual oder pauschal erheben müssen, wenn sie ihre Ausgaben mit den Zuweisungen aus dem Gesundheitsfonds nicht decken können. Erhalten die Kassen aus dem Fonds mehr, als sie für die Gesundheitsversorgung ihrer Versicherten benötigen, können sie die überschüssigen Mittel an ihre Mitglieder ausschütten. Zur Verhinderung einer finanziellen Überforderung der Kassenmitglieder durch die Zusatzbeiträge, ist eine 1\%-Überforderungsklausel vorgesehen, die sich am beitragspflichtigen Einkommen orientiert. Bei pauschalen Zusatzbeiträgen bis 8 Euro gilt diese Klausel jedoch nicht.

Damit der künftige Beitragswettbewerb funktioniert, müssen folgende Anforderungen erfüllt sein: Die GKVMitglieder müssen Beitragsunterschiede wahrnehmen und preissensibel reagieren, und die Zusatzbeiträge müssen möglichst unverzerrte Preissignale aussenden.

2009, im Startjahr des Gesundheitsfonds, soll dieser finanziell so ausgestattet sein, dass alle GKV-Ausgaben gedeckt werden. In dieser Situation kann man davon ausgehen, dass einige Kassen einen Zusatzbeitrag erheben müssen, andere dagegen Ausschüttungen vornehmen können. In Bezug auf die Transparenz von Beitragsunterschieden werden die GKV-Mitglieder insoweit vor allem wahrnehmen, ob eine Kasse einen Zusatzbeitrag erhebt oder nicht bzw. ob es sogar Kassen gibt, die finanzielle Mittel ausschütten. Vor diesem Hintergrund werden Kassen extrem darum bemüht sein, solange wie irgend möglich ohne Zusatzbeitrag auszukommen. Das dürfte mit steigendem Ausgabenniveau jedoch für immer weniger Kassen möglich sein, weil die allgemeine Deckung durch den Fonds auf $95 \%$ der GKV-Ausgaben zurückgefahren wird. Damit werden mehr und mehr Kassen in die Situation geraten, einen Zusatzbeitrag erheben zu müssen. Überdies erscheint es keineswegs unrealistisch, dass die 95 \%-Deckung der GKV-Ausgaben durch den Gesundheitsfonds fällt. Sonst müsste entweder der einheitliche Beitragssatz zum Fonds oder der Zufluss von Steuermitteln erhöht werden - beides für die Politik nicht gerade erstrebenswerte Aussichten.

Wenn mehr oder weniger alle Kassen einen Zusatzbeitrag erheben, müssen die Mitglieder preissensibel auf Beitragsdifferenzen reagieren, damit der Beitragswettbewerb über den Parameter „Zusatzbeitrag“ funktioniert. In diesem Zusammenhang ist die 1\%-Überforderungsklausel von Bedeutung, denn sie kann zu preisunsensiblen Wahlentscheidungen bei allen GKV-Mitgliedern führen, die entweder grundsätzlich bereit sind, $1 \%$ ihres Einkommens als Zusatzbeitrag zu zahlen, oder die dazu gezwungen sind, weil sie keine Kasse mehr finden, die einen Zusatzbeitrag verlangt, der weniger als 1\% ihres Einkommens beträgt. Wer etwa bei einem beitragspflichtigen Einkommen von 1.500 Euro pro Monat bereit ist, 15 Euro als Zusatzbeitrag zu zahlen, oder wer keine Kasse mehr findet, die weniger als 15 Euro Zusatzbeitrag verlangt, kann ohne finanzielle Einbuße auch eine Kasse wählen, die 20 oder 30 Euro Zusatzprämie fordert. Solange sich die $1 \%$-Überforderungsklausel auf den individuellen Zusatzbeitrag der gewählten Kasse bezieht, tritt somit 
ein Problem auf, das es ähnlich bereits bei den Gesundheitsprämienmodellen gab und das dort durch die Anbindung der Belastungsgrenze an eine Durchschnittsprämie gelöst wurde. Weil eine vergleichbare Regelung nunmehr fehlt, kann die 1 \%-Überforderungsklausel dazu führen, dass es für einen nennenswerten Teil der GKV-Mitglieder gar keine Beitragsunterschiede mehr gibt - Intensivierung des Wettbewerbs?

Besonders krude wirkt die Regelung, dass die 1\%-Überforderungsklausel bei einem Zusatzbeitrag von bis zu 8 Euro generell nicht greift. Zum einen sind natürlich die Verteilungswirkungen problematisch, wenn in diesem Fall ausgerechnet von besonders einkommensschwachen Mitgliedern mit beitragspflichtigen Einkommen bis zu 800 Euro ein höherer Anteil des Einkommens für den Zusatzbeitrag aufgewendet werden muss, als es „eigentlich“ vorgesehen ist, um finanzieller Überforderung vorzubeugen. Ein Mitglied mit einem Einkommen von 400 Euro im Monat müsste bei einem Zusatzbeitrag von 8 Euro immerhin $2 \%$ seines Einkommens hierfür aufwenden. Allerdings können Geringverdiener ihre Belastung senken und der skizzierten Lage entkommen. Einerseits natürlich dadurch, dass sie eine Kasse wählen, die einen niedrigeren oder gar keinen Zusatzbeitrag erhebt ${ }^{4}$. Freilich setzt dies voraus, dass es solche Kassen am Markt überhaupt noch gibt. Ist dies nicht der Fall, ist es dagegen vorteilhaft, in eine Kasse mit einem Zusatzbeitrag von mehr als 8 Euro zu wechseln, weil in diesem Fall die $1 \%$-Überforderungsklausel greift und das Mitglied mit einem Einkommen von 400 Euro nur 4 Euro entrichten muss. Im Hinblick auf wettbewerbspolitische Zielvorstellungen kann eine solche Anreizstruktur nur als absurd bezeichnet werden.

Zudem kann die 1\%-Überforderungsklausel beim Zusatzbeitrag zu nennenswerten Verzerrungen des Beitragswettbewerbs führen, da sie für Kassen mit vielen einkommensschwachen Mitgliedern zu einem gravierenden Einnahmeproblem führt. Wenn viele Mitglieder einer Kasse unter die $1 \%$-Überforderungsklausel fallen, zahlen diese Mitglieder nicht mehr den vollen Zusatzbeitrag, und die Kasse nimmt weniger ein, als sie benötigt. Sie muss ihren Zusatzbeitrag entsprechend erhöhen, damit der „Fehlbetrag" durch die übrigen Mitglieder ausgeglichen wird sofern diese bei einem höheren Zusatzbeitrag nicht auch unter die Überforderungsklausel fallen. Wenn schließlich kein Mitglied mehr da ist, das den erforderlichen Zusatzbeitrag vollständig bezahlt, muss die Kasse schließen aber nicht etwa, weil sie weniger wirtschaftlich handeln würde als andere Kassen, sondern allein, weil sie viele einkommensschwache Mitglieder versichert, von denen sie die erforderlichen Zusatzbeiträge wegen der Überforderungsklausel gar nicht einziehen kann. Ein wirksames Preissignal kann der Zusatzbeitrag unter diesen Bedingungen wahrlich nicht aussenden - genau dies wäre jedoch die Voraussetzung für einen funktionierenden Beitragswettbewerb.

Der Umgang mit diesem gravierenden Funktionsdefizit des neuen Finanzierungsmodells „Gesundheitsfonds plus
Zusatzbeitrag" wirft zudem ein bezeichnendes Licht auf das fachliche Niveau, das beim Zustandekommen der Gesundheitsreform geherrscht hat. Die Politik hat das skizzierte Problem nämlich sehr wohl gesehen und zu seiner Lösung sogar eigens zwei gutachterliche Stellungnahmen in Auftrag gegeben. Beide Gutachter - Eckart Fiedler und Bert Rürup - haben die massiven Wettbewerbsverzerrungen bei einem Zusatzbeitrag ohne kassenübergreifenden Einkommensausgleich bestätigt und entsprechende Lösungsvorschläge vorgelegt, die jedoch im weiteren Gesetzgebungsprozess - zumindest bis zum Fraktionsentwurf - keine weitere Beachtung fanden. Wie schon bei den weiterhin ungelösten Finanzierungsproblemen der GKV (Stichwort: Wachstumsschwäche der beitragspflichtigen Einnahmen) erfolgt somit auch hier statt einer gezielten Problemlösung die vollständige Ignoranz des Problems.

\subsection{Unvollständiger Morbi-RSA}

Aber nicht nur Einkommensselektion wird künftig ein prägendes Kennzeichen des Kassenwettbewerbs sein, sondern aller Voraussicht auch weiterhin Selektion der Versicherten nach Morbiditätskriterien. Was bislang in Bezug auf den künftigen Schlüssel zur Verteilung der Fondsmittel auf die einzelnen Kassen im Gesetzentwurf vorgesehen ist, lässt dies zumindest befürchten. Für diese Einschätzung sprechen vor allem zwei Gründe:

- Zum einen soll das Spektrum der künftig im Risikostrukturausgleich (RSA) gesondert berücksichtigten Krankheiten auf „50 bis 80 insbesondere kostenintensive chronische Krankheiten und Krankheiten mit schwerwiegenden Verlauf" begrenzt sein. Eine derartige Zahl als Ergebnis eines politischen Verhandlungsprozesses ohne erkennbaren Bezug zu empirischen Ergebnissen wissenschaftlicher Analysen in einem Gesetz vorzugeben, bedeutet ein hohes Maß an Willkür, was im Hinblick auf eine möglichst funktionale Sachlösung von vornherein skeptisch stimmen muss.

Dabei fangen die Unklarheiten - und entsprechend die möglichen Anknüpfungspunkte für weitere willkürliche Festlegungen bei der Konkretisierung und Umsetzung des beschlossenen Morbiditätskonzepts - schon bei der Definition dessen an, was überhaupt unter dem Begriff „Krankheit" verstanden werden soll. Im Gesetzentwurf heißt es in Artikel 30 zur Änderung der RisikostrukturAusgleichsverordnung, dass „die Krankheiten (...) eng abgegrenzt sein (sollen)“ - ein Indiz dafür, dass eher nur ein begrenzter Ausschnitt aus dem gesamten Krankheitspanorama in Zukunft bei der Verteilung der Fondsmittel gesondert berücksichtigt werden soll? Im Gutachten von IGES/Lauterbach/Wasem hatte es dagegen - entsprechend dem unstrittigen wissenschaftlichen Erkenntnisstand in dieser Frage - geheißen: „Klassifikationsmodelle, die nicht das gesamte Morbiditätsspektrum berücksichtigen, lassen notwendigerweise mehr Selektionsspielraum als ,morbiditätsvollständige' Modelle." (IGES/Lauterbach/Wasem 2004, S. 91). 
- Neben der willkürlichen Begrenzung der Zahl zu berücksichtigender Krankheiten im künftigen RSA - bei überdies noch ungeklärtem Krankheitsbegriff - lässt zum anderen ein weiteres Merkmal des vereinbarten Ausgleichsmechanismus befürchten, dass das Ziel einer möglichst vollständigen Berücksichtigung von Morbiditätsunterschieden bei der Verteilung der Fondsmittel auf die Kassen - unabdingbare Voraussetzung für einen unverzerrten und auf die Verbesserung von Qualität und Wirtschaftlichkeit der Gesundheitsversorgung abzielenden Kassenwettbewerbs - deutlich verfehlt werden könnte. So heißt es nämlich im Gesetzentwurf zum GKV-WSG (Artikel 38 Nr. 6, § 31 Abs. 1), dass insbesondere solche Krankheiten gesondert berücksichtigt werden sollen, „bei denen die durchschnittlichen Leistungsausgaben je Versicherten die durchschnittlichen Leistungsausgaben aller Versicherten um mindestens 50 vom Hundert übersteigen". Unabhängig davon, dass der vorgesehene 50-Prozent-Schwellenwert vollkommen willkürlich ist (wie es jeder andere Schwellenwert auch wäre), ist diese Formulierung nicht nur interpretationsfähig, sondern sie ist interpretationsbedürftig, wenn ein konkretes Klassifikationsmodell ausgearbeitet wird, was dem Gesetzentwurf zufolge bis zum 31.10.2007 durch ein Gutachten eines eigens beim Bundesversicherungsamt eingesetzten wissenschaftlichen Beirats erfolgen soll.

Die fragliche Formulierung im Gesetzentwurf kann so verstanden werden, dass nur solche Krankheiten gesondert berücksichtigt werden sollen, deren spezifische Kosten - ermittelt als krankheitsspezifischer Zuschlag im Rahmen eines Regressionsmodells - den geforderten Schwellenwert von 50 Prozent der durchschnittlichen Leistungsausgaben aller Versicherten übersteigen. Diese Lesart würde bedeuten, dass insbesondere für viele multimorbide Versicherte keine gesonderten Morbiditätszuschläge geleistet würden, weil diese Versicherten zwar in der Gesamtheit aller ihrer Erkrankungen hohe Versorgungskosten weit über dem genannten Schwellenwert verursachen, diese Bedingung aber in isolierter Betrachtung unter Umständen für keine der im einzelnen vorliegenden Krankheiten erfüllt wird. Diese Interpretation, die insbesondere viele ältere, multimorbide Versicherte aus der künftigen Morbiditätsorientierung bei der Verteilung der Fondsmittel auf die einzelnen Kassen „ausblendet", würde vermieden, wenn die Interpretation der geplanten Gesetzesvorschrift anders vorgenommen würde, nämlich - weitgehend analog zur gegenwärtigen Berücksichtigung von eingeschriebenen Disease-ManagementVersicherten im RSA - so, dass Krankheiten dann gesondert berücksichtigt werden, wenn die Gesamtausgaben der Versicherten mit diesen Krankheiten den geforderten 50-Prozent-Schwellenwert überschreiten.

In jedem Fall sind die vorgesehenen Gesetzesformulierungen unpräzise und auslegungsfähig bzw. -bedürftig; spätestens wenn das vorgesehene Gutachten zur Auswahl der berücksichtigungsfähigen Krankheiten im Herbst 2007 vorliegt, dürften sich die entsprechenden Kontroversen fortsetzen. Für die Versorgung multimorbider Ver- sicherter mit mehreren chronischen Erkrankungen ist dies in jedem Fall eine schlechte Perspektive, denn solange nicht hinreichend gesichert ausgeschlossen ist, dass diese Versicherten in Zukunft kein „schlechtes Risiko“ für ihre Krankenkasse darstellen, kann keine Kasse ein besonderes Interesse haben, sich gerade um diese Klientel mit besonderer Aufmerksamkeit zu kümmern - dies sollte mit der Morbiditätsorientierung des RSA aber gerade bezweckt werden. Auch in dieser Frage hat die Koalition letztlich politisches Feilschen vor Sachverstand gesetzt, und schon jetzt scheint gewiss: Die im Konsenspapier der Koalitionsparteien von Anfang Oktober 2006 aufgestellte Behauptung, dass „Krankenkassen mit einer hohen Zahl überdurchschnittlich kranker Versicherter (...) künftig keine Nachteile im Wettbewerb mehr (haben)" (Konsenspapier: Zentrale Inhalte der Gesundheitsreform 2006, S. 3), wird in dieser Absolutheit ganz sicher nicht zutreffen.

\section{Zwischenfazit:}

Die Autonomie der Beitrags-(Preis-)Setzung wird den Kassen durch die Einrichtung des zentralen Gesundheitsfonds weitgehend genommen, denn der Beitragssatz wird künftig einheitlich per Rechtsverordnung festgelegt. Unterschiede beim kassenspezifischen Zusatzbeitrag werden voraussichtlich für immer mehr GKV-Mitglieder aufgrund der $1 \%$-Überforderungsklausel gar nicht mehr finanziell spürbar sein; zudem besteht die Gefahr, dass Kassen mit vielen Einkommensschwachen durch diese Regelung in die unvermeidliche Insolvenz getrieben werden. Die unvermeidliche Folge sind massive Anreize der Kassen zu einer Mitgliederselektion nach Einkommen, die aufgrund eines vermutlich unzureichenden MorbiRSA durch eine fortgesetzte Versichertenselektion nach Morbidität begleitet wird. Die im Gesetzentwurf vorgesehenen neuen Finanzierungsstrukturen bieten somit keinesfalls rosige Wettbewerbsperspektiven!

\section{Die Leistungsseite: Neuregelungen für die Vertragsbeziehungen zwischen Kassen und Leistungsanbietern}

Wenn sich Kassen im Wettbewerb dadurch profilieren sollen, dass sie für Versicherte - und zwar unabhängig von deren Einkommen, Alter, Geschlecht oder Morbiditätsstatus - eine qualitativ gute und effizient erbrachte Versorgung bieten, genügt es allerdings nicht, dass die finanziellen Anreize auf dieses Ziel ausgerichtet sein müssen. Es ist ebenfalls erforderlich, dass genügend wettbewerbliche Instrumente zur Verfügung stehen, mit denen die Kassen in einen echten Preis-Leistungs-Wettbewerb treten können. Bei einem weithin einheitlichen Leistungskatalog und gleichen Mindestqualitätsstandards betrifft dies insbesondere die Art und Weise der Leistungserstellung und deren Vergütung. Nur so können zwischen den Kassen überhaupt tatsächliche Unterschiede in der Wirtschaftlichkeit der Versorgung entstehen. Ein unverzerrter und funktionsfähiger Leistungswettbewerb ist somit ein notwendiges Pendant zur bereits vor 10 Jahren eingeführten Kassenwahlfreiheit ${ }^{5}$; er erfordert zwin- 
gend hinreichende Handlungsfreiheiten in den Vertragsbeziehungen zwischen Kassen und Leistungserbringern auf einer dezentralen Verhandlungsebene.

Bisher läuft der Kassenwettbewerb um Versicherte genau an diesem Punkt weitgehend ins Leere. Zwar gibt es inzwischen einige Möglichkeiten zu selektivem Kontrahieren, doch sind diese vor allem für die Kassen zu wenig anreizkompatibel ausgestaltet und werden deshalb - mit Ausnahme der integrierten Versorgung - kaum genutzt ${ }^{6}$. Ein „Wettbewerbsstärkungsgesetz“ weckt natürlich hohe Erwartungen, dass endlich erkennbar wettbewerbsorientierte Reformen in Bezug auf die Vertragsbeziehungen zwischen Krankenkassen und Leistungserbringern erfolgen. $\mathrm{Ob}$ diese Erwartungen tatsächlich erfüllt werden, lässt sich jedoch gar nicht so einfach feststellen, denn einmal mehr werden wieder nur punktuell hier und da wettbewerbliche Öffnungen in das ansonsten unverändert plan- und kollektivwirtschaftliche Leistungserbringungsrecht eingestreut, so dass sich schon von vornherein sagen lässt: Eine generelle wettbewerbliche Öffnung im Sinn der Einführung eines konsistenten vertragswettbewerblichen Systems mit Selektivverträgen sieht anders aus ${ }^{7}$.

\subsection{Antiwettbewerbliche Reformelemente}

Offenbar ist eine generelle vertragswettbewerbliche Öffnung aber auch gar erwünscht, denn sonst wäre es kaum möglich, dass der Gesetzentwurf sogar Regelungen vorsieht, die explizit das diametrale Gegenteil einer solchen Öffnung bedeuten. Das gilt insbesondere für die bisherige Selektivvertragsoption für hochspezialisierte ambulante Leistungen im Krankenhaus (§ 116b SGBV). Diese Selektivvertragsoption wurde von den Krankenkassen bisher aus zwei einfachen Gründen nicht genutzt: Zum einen führt ein solcher Selektivvertrag aufgrund fehlender Bereinigungsvorschriften zu einer „Add-onFinanzierung“ zu den bestehenden Kollektivvergütungen, also zu Mehrausgaben, woran keine im Wettbewerb stehende Kasse interessiert sein kann. Zum anderen sind Versorgungsverträge für Schwerstkranke ohne einen morbiditätsorientierten Risikostrukturausgleich mit einem hohen finanziellen Zusatzrisiko verbunden. Die mangelnde Anreizkompatibilität dieser Selektivvertragsoption ist somit offensichtlich.

War daher prinzipiell folgerichtig noch in den ersten Arbeitsentwürfen zum GKV-WSG vorgesehen, die finanzielle Anreizkompatibilität für die Kassen durch eine wenngleich etwas krude ausgestaltete - 0,5 \%-Anschubfinanzierung zu erhöhen, wenden sich die Verfasser des Gesetzentwurfs inzwischen einem ihnen wesentlich vertrauteren Steuerungsinstrument zu. Den Kassen wird die Vertragskompetenz entzogen, und die Versorgung nach „bewährtem“ plan- und kollektivwirtschaftlichen Muster geregelt: Der Gesetzgeber bestimmt die Leistungen und Krankheiten, die Krankenhäuser ambulant erbringen dürfen, und die Krankenhausplanungsinstanzen der Länder legen fest, welche Krankenhäuser diese Leistungen ambulant erbringen und abrechnen dürfen. Den Kassen bleibt nur noch, die erbrachten Leistungen zu bezahlen, und zwar zunächst auf der Basis eines einheitlich und gemeinsam festgelegten durchschnittlichen Punktwerts, ab 01.01.2009 dann nach einer in der jeweiligen Region einheitlich geltenden Euro-Gebührenordnung. Die ehemals wettbewerbsorientiert angelegte Selektivvertragsoption wird damit vollständig „entwettbewerblicht“ und in eine neue Kollektivregelung mit Kontrahierungszwang und Einheitspreisen für alle Kassen überführt ${ }^{8}$.

Für den gesamten Krankenhaussektor ist dieses Steuerungskonzept jedoch „normal“. Während dem Gesetzentwurf zufolge im vertragsärztlichen Bereich für „besondere ambulante Versorgung" (§ 73c SGB V) eine inhaltlich vergleichsweise weit gefasste Selektivvertragsoption eröffnet werden soll, sind analoge Regelungen im Krankenhaussektor nicht vorgesehen. Ausgerechnet im ausgabenstärksten Leistungsbereich der GKV soll somit auch künftig gerade nicht auf vertragswettbewerbliche Steuerung gesetzt werden. Dabei läge gegenüber dem geplanten klassischen Kostendämpfungsvorhaben, die Krankenhäuser über eine staatlich verordnete Preissenkung in Form eines generellen 1\%-Sanierungsbeitrags zur Stabilisierung der GKV-Ausgaben heranzuziehen, gerade in größeren Vertragsfreiräumen eine deutlich sinnvollere Alternative.

Inwieweit die einstweilen lediglich angekündigte Anpassung des Ordnungsrahmens der Krankenhausversorgung Impulse in Richtung Wettbewerbsstärkung geben kann, bleibt abzuwarten, insbesondere ob es tatsächlich dazu kommt, dass notwendige Veränderungen bei der Krankenhausplanung (Stichwort: Leistungsmengen- statt Bettenplanung) und der dualen Finanzierung vorgenommen werden. Die jetzt erst einmal vorgenommene Stärkung der Position der Krankenhausplanungsinstanzen der Länder lässt hier wenig Positives erwarten. Und von individuellen Vergütungsverhandlungen zwischen Kassen und Krankenhäusern ist ohnehin erst gar nicht die Rede - hier werden nach dem Ende der Konvergenzphase erst einmal regionale Einheitspreise gelten.

Diese Erwartung wird dadurch genährt, dass der Gesetzentwurf bei der vertragsärztlichen Vergütung in dieselbe Richtung weist, denn dort sollen auf regionaler Ebene ebenfalls Einheitspreise eingeführt werden. Ein Vergütungssystem, das vollständig außerhalb des Verhandlungsbereichs der einzelnen Kassen liegt und in Einheitspreise für die vertragsärztliche Versorgung mündet, hat mit Wettbewerb nichts zu tun. Ebenso wenig wie die Regelung, den einzelnen Kassen einerseits das Morbiditätsrisiko zu übertragen, ohne ihnen jedoch andererseits vertragliche Gestaltungsfreiräume zu geben, um steuernd auf die Ausgaben einwirken zu können, die mit dem Morbiditätsrisiko verbunden sind.

\subsection{Reformierte Selektivvertragsoptionen}

Wie bereits festgestellt, enthält der Gesetzentwurf keine generelle vertragswettbewerbliche Öffnung für Selektivverträge, sondern sieht lediglich Erweiterungen des be- 
stehenden „Flickenteppichs von Partikularlösungen“ vor. Damit geht unweigerlich die Gefahr von Intransparenz, Unvollständigkeiten und vor allem Inkonsistenzen einher - und zwar sowohl hinsichtlich der wechselseitigen Abstimmung unterschiedlicher Selektivvertragsoptionen als auch im Hinblick auf den Koordinationsbedarf gegenüber den Regelungen des jeweiligen Kollektivvertragssystems. Dies betrifft insbesondere drei generelle Aspekte von Selektivvertragsoptionen, die letztlich entscheidende Faktoren dafür sind, ob das Wettbewerbspotenzial tatsächlich ausgeschöpft wird:

- die konsequente Bereinigung der Kollektivvergütungsansprüche,

- der Verzicht auf einschränkende Detailregulierungen für Vertragsinhalte sowie

- Möglichkeiten zur freiwilligen Bindung von Versicherten oder Leistungserbringern an die Selektivvertragspartner einer Kasse.

\section{Bereinigungen der Kollektivvergütungsansprüche}

Wenn Selektivverträge neben das Kollektivsystem treten und zu einer effizienteren Versorgung beitragen sollen, ist die Bereinigung der kollektiven Vergütungsansprüche ein notwendiger Bestandteil der wettbewerblichen Rahmenordnung. Die Bereinigung der Kollektivvergütungen (vertragsärztliche Gesamtvergütungen, Krankenhausbudgets) muss dabei um jene Leistungen erfolgen, die nicht mehr im Rahmen der Kollektivverträge, sondern nunmehr selektivvertraglich abgedeckt sind. Dabei muss der Bereinigungsbetrag daran orientiert sein, was die nun selektivvertraglich erbrachten Leistungen ansonsten im Kollektivsystem gekostet hätten.

Hinter den Anforderungen einer konsequenten Bereinigung steht die ökonomische Grundlogik, dass die im Beitragswettbewerb stehenden Kassen kein Interesse daran haben können, für bestimmte Leistungen doppelt zu bezahlen. Zugleich würde durch eine konsequente Bereinigung auch die Anreizkompatibilität von Selektivverträgen für Leistungserbringer erhöht, weil dadurch die Einkommenschancen im Kollektivsystem sinken, bei Selektivverträgen aber spürbar steigen können, da den Kassen eine gesicherte Finanzierung dieser Verträge möglich ist.

Zwar wird die Bereinigungsproblematik für Selektivverträge zur hausarztzentrierten Versorgung, zur besonderen ambulanten Versorgung und zur integrierten Versorgung im Gesetzentwurf durchaus erkannt, aber nach wie vor nicht hinreichend zielgenau, praktikabel und zwingend geregelt. Der vorgesehene Bereinigungsansatz - eine Verhandlungslösung, bei der die Kollektivvertragspartner über die Bereinigung verhandeln müssen - hat sich bereits in der Vergangenheit als untauglich erwiesen. Er wird sich bei der vorgesehenen Stärkung des „Gemeinsam und Einheitlich“ in der vertragsärztlichen Versorgung sogar künftig noch problematischer darstellen als bereits zurzeit. Damit eine Kasse für selektivvertraglich vereinbarte Leistungen Finanzmittel aus der Gesamtver- gütung abziehen darf, bedarf es nämlich nicht mehr nur der Zustimmung der Kassenärztlichen Vereinigung (die hieran naturgemäß kein Interesse haben kann), sondern es müssen auch die anderen Kassen gemeinsam und einheitlich zustimmen. Damit können Mitbewerber zentrale Rahmenbedingungen des Vertragshandelns ihrer Konkurrenten unmittelbar beeinflussen.

Notwendig wäre eine klare, schiedsamts- und schiedsstellenfähige Bereinigungsregelung im Sinn einer verpflichtenden Bereinigungsvorgabe für die Kollektivvertragspartner, die nicht mehr von deren Einverständnis abhängig wäre. Damit müssten Kassen, die entsprechende Selektivverträge vorweisen können, die Bereinigung direkt von den Kollektivvertragspartnern einfordern können. Durch eindeutige und präzise Bereinigungsvorgaben würde im Streitfall auch die Handlungs-

fähigkeit der anzurufenden Schiedsämter bzw. Schiedsstellen deutlich erhöht.

Auch wenn die pauschale Anschubfinanzierung vor allem längerfristig keinen Ersatz für konsequente und sachgerechte Bereinigungsregelungen darstellen kann, hat sich die pragmatische Einstiegslösung des 1\%-Budgetabzugs zur "Anschubfinanzierung“ in der integrierten Versorgung als ausgesprochen wirksam für den Abschluss von Selektivverträgen erwiesen. Nicht zuletzt aus diesem Grund sind von Krankenkassen auch andere Selektivvertragsmodelle - etwa zur hausarztzentrierten Versorgung im Rahmen der Regelungen zu integrierten Versorgung umgesetzt worden. Deshalb wäre es hilfreich gewesen, das Instrument der Anschubfinanzierung nicht auf die integrierte Versorgung zu beschränken, sondern auch anderen Selektivvertragsoptionen zugänglich zu machen. Allerdings würde auch dieser Schritt grundsätzlich nichts daran ändern, dass Selektivverträge als effizienzförderndes Substitut von Teilen der kollektivvertraglich geregelten Versorgung eindeutige und verpflichtende Bereinigungsvorgaben für die bestehenden kollektivvertraglichen Vergütungsansprüche erfordern. Gibt es diese verpflichtenden Bereinigungsvorgaben weiterhin nicht, bleibt das Gerede von wettbewerblichen Handlungsmöglichkeiten der Kassen zur Erhöhung der Versorgungseffizienz bloße Rhetorik.

\section{Detailvorgaben für Selektivverträge}

In der integrierten Versorgung hat sich der pragmatische Pauschalabzug zur Anschubfinanzierung als hochgradig anreizkompatibel für eine lebhafte Umsetzung von Selektivverträgen erwiesen. Dennoch - oder deshalb? - scheint die beträchtliche wettbewerbliche Eigendynamik in diesem Bereich den Verfassern des Gesetzentwurfs offenbar aber schon wieder zu weit bzw. in die falsche Richtung zu gehen. Daher soll die Nutzung der Anschubfinanzierung künftig daran geknüpft werden, dass es sich um Verträge mit „bevölkerungsbezogener Flächendeckung" handelt - was immer das wirklich konkret sein soll. Angesichts der Begehrlichkeiten in Bezug auf die begrenzten Mittel der Anschubfinanzierung werden sich gewiss bald Sozialgerichte damit befassen müssen, ob ein 
Vertrag dem gesetzlich präferierten Typus von integrierter Versorgung entspricht oder nicht.

In der Tat beziehen sich die meisten bestehenden Integrationsverträge auf eher kleinteilig abgegrenzte Versorgungsaufgaben, während populationsbezogene (Voll)Versorgungsmodelle bislang nur relativ selten in Angriff genommen wurden. Auch hierfür gibt es eindeutige Ursachen in Form von unzureichender Anreizkompatibilität der bestehenden gesetzlichen Regelungen. Neben dem ungelösten Bereinigungsproblem - jenseits und nach der Ausschöpfung der 1\%-Anschubfinanzierung zählt dazu vor allem die unzureichende Investitionssicherheit für die Entwicklung und den Aufbau umfassender integrierter Versorgungsmodelle. Anstatt dieses Problem jedoch anreizkompatibel zu lösen, indem eindeutige und verbindliche Bereinigungsvorschriften außerhalb des Einflussbereichs der Kollektivvertragspartner erlassen oder aber zumindest der pragmatische Pauschalabzug der Kollektivvergütungen zeitlich deutlich verlängert ${ }^{9}$ und vom Volumen her erhöht würde, soll die Verwendung der Anschubfinanzierung künftig nur bestimmten Versorgungsformen vorbehalten bleiben. Obwohl noch kein einziger Integrationsvertrag einigermaßen verlässlich evaluiert ist, verfügt der Gesetzgeber offenbar schon über hinreichendes Wissen, den Selektivvertragspartnern detaillierte Vorgaben über die inhaltliche Ausrichtung der Vertragsgestaltung zu machen.

Dieses Vorgehen wirft ein bezeichnendes Licht auf das derzeitige Rollenverständnis des Gesetzgebers. Wenn die Wettbewerbsrhetorik ernst gemeint wäre und wettbewerbliche Such- und Entdeckungsprozesse im Leistungsmarkt der GKV tatsächlich umfassend gestärkt werden sollten, müsste der Gesetzgeber auf prozesspolitische Eingriffe und Detailregulierungen zur Ausgestaltung selektivvertraglicher Versorgungsformen vollständig verzichten. Dass hiervon jedoch keine Rede sein kann, zeigt sich auch bei einer anderen Selektivvertragsoption. So soll die Ausbreitung von hausarztzentrierter Versorgung auf dirigistischem Weg über eine Angebotspflicht der Kassen bewirkt werden, statt auf Mechanismen der wettbewerblichen Anreizsteuerung zu setzen, deren unzureichende Ausprägung bislang für die eher schleppende Umsetzung dieser Selektivvertragsoption verantwortlich war.

Immerhin bleibt hier wenigstens - im Unterschied zur hochspezialisierten ambulanten Krankenhausbehandlung - der Selektivvertragscharakter erhalten. Wie „selektiv“ die Verträge zur hausarztzentrierten Versorgung angesichts der Vorgabe der Flächendeckung am Ende tatsächlich ausfallen werden, kann nur gemutmaßt werden. Es ist zu befürchten, dass am Ende vor allem (kassenindividuelle) Kollektivverträge mit großen Hausärzteverbänden oder Kassenärztlichen Vereinigungen geschlossen werden, die zwar kaum eine Ausschöpfung von Wirtschaftlichkeitspotenzialen versprechen, aber der Anforderung der Flächendeckung genügen.

Ohnehin werden Kassen und Leistungserbringer die Ausgestaltung der hausarztzentrierten Versorgung nur sehr eingeschränkt im Wettbewerb nutzen können, denn der Gesetzentwurf enthält - über die Vorgabe der Flächendeckung hinaus - weit reichende Vorschriften darüber, wie die hausarztzentrierte Versorgung auszusehen hat. So wird etwa genau vorgeschrieben, welche Anforderungen die teilnehmenden Ärzte erfüllen müssen. Auch hier verfügt der Gesetzgeber offenbar bereits über hinreichend präzises Wissen, das wettbewerbliche Suchprozesse von vornherein überflüssig macht. Das augenscheinlich unveränderte Rollenverständnis des Gesetzgebers als detaillistischer Prozessregulator steht allerdings auch hier im grundsätzlichen Widerspruch zu einer Stärkung wettbewerblicher Steuerungsmechanismen und der entsprechenden Wettbewerbsrhetorik.

\section{Bindungsmöglichkeiten von Versicherten und Leistungserbringern}

Wettbewerbsteuerung wirklich nutzen zu wollen, heißt auch, Vertrauen darauf zu setzen, dass sich durch freie Angebotsentscheidungen von Krankenkassen und Leistungserbringern und freie Nachfrageentscheidungen von Versicherten am Ende solche Versorgungsmodelle durchsetzen, die eine bedarfsgerechte, qualitativ hochwertige und wirtschaftliche Versorgung bieten. Gerade dann werden Versicherte auch bereit sein, sich freiwillig an die Einhaltung bestimmter Teilnahmebedingungen zu binden. Dazu gehört die Festlegung, im Regelfall nur bestimmte Leistungsanbieter aufzusuchen oder zum Beispiel die Bindung von Ärzten, nur bestimmte Medikamente zu verordnen. Solche Bindungsmöglichkeiten sind unverzichtbarer Bestandteil einer konsequenten Wettbewerbsstärkung über Selektivvertragsoptionen, denn erst entsprechende Bindungsmöglichkeiten verschaffen den Selektivvertragspartnern hinreichende Planungs- und Kalkulationssicherheit -nicht zuletzt als Voraussetzung für Preis- bzw. Rabattverhandlungen.

Die Notwendigkeit von Bindungsoptionen an bestimmte Selektivvertragspartner der Kassen wurde auch von den Verfassern des Gesetzentwurfs in vielen Bereichen richtig erkannt. So ist im Gesetzentwurf zum GKVWSG die Möglichkeit der Versichertenbindung bei der hausarztzentrierten Versorgung, bei der besonderen ambulanten Versorgung, bei der integrierten Versorgung und in der Hilfsmittelversorgung vorgesehen. Leider ist sie nach wie vor allgemein für Arzneimittel, außer im Rahmen hausarztzentrierter oder integrierter Versorgung, nicht möglich. Hier hätte die Option, Leistungsanbieter auf ein bestimmtes Verordnungsverhalten verpflichten zu können - etwa über kassenspezifische Positivlisten ${ }^{10}$ dazu beitragen können, die Rabattpotenziale in den Verträgen zwischen Kassen und Arzneimittelherstellern mit Leben zu füllen. Dass die neu geschaffenen Rabattoptionen in Bezug auf Verhandlungen zwischen Kassen und Apothekern bei unverändert fortbestehendem Fremdund Mehrbesitzverbot große Wirkungen entfalten, erscheint ohnehin unwahrscheinlich.

Allein im Hilfsmittelbereich sieht der Gesetzentwurf einen konsequenten Übergang zu Selektivvertragsmodellen vor. Versicherte können an bestimmte Hilfsmittelliefe- 
ranten gebunden werden, wodurch sich der Verhandlungsspielraum für Preisabschläge von Hilfsmittelanbietern spürbar erhöhen dürfte. Kassen können den Hilfsmittelanbieter nunmehr im Gegenzug für Rabatte auch bestimmte Absatzmengen zusichern. Somit gibt es wenigstens eine - kleine - Versorgungsnische, in der der Gesetzentwurf dem Anspruch der effizienzfördernden Wettbewerbsstärkung wirklich gerecht wird.

\section{Fazit}

Was kann nun zusammenfassend vom „GKV-Wettbewerbsstärkungsgesetz" für die Wettbewerbsstärkung in der GKV erwartet werden?

Die geplanten Finanzierungsstrukturen, „Gesundheitsfonds plus Zusatzbeitrag“, werden nicht nur das Ziel einer stabilisierten und gerechteren Finanzierung verfehlen, sie können zudem zu nennenswerten Verzerrungen des künftigen Beitragswettbewerbs führen. Aufgrund der $1 \%$-Überforderungsklausel werden Beitragsunterschiede voraussichtlich für immer mehr GKV-Mitglieder gar nicht mehr finanziell spürbar sein. Den Kassen werden Anreize gesetzt, ihre wettbewerblichen Aktivitäten auf möglichst einkommensstarke Mitglieder und - wahrscheinlich noch immer - zugleich möglichst gesunde Versicherte zu fokussieren. Hier könnten die neuen Wahltarife für Selbstbehalte und Schadenfreiheitsrabatte das passende Instrumentarium bieten. Diese Tarife sind von ihrem Grundprinzip her vor allem für Gesunde interessant und werden aufgrund der vorgesehenen einkommensabhängigen Prämienausschüttung vor allem einkommensstarke Gesunde ansprechen.

Die leistungsseitigen Reformvorhaben des Gesetzentwurfs bieten wenig Substanzielles im Hinblick auf erweiterte Vertragsfreiheiten für Kassen und Leistungsanbieter. Eine generelle vertragswettbewerbliche Öffnung für sektorale wie sektorübergreifende Selektivverträge ist nicht vorgesehen.

Im Strauß der Selektivvertragsoptionen finden sich nun zusammengefasst u.a. Maßnahmen, die zwar in Richtung mehr Vertragswettbewerb weisen sollen, die allerdings nicht ausreichend durchdacht sind und damit das wettbewerbliche und effizienzfördernde Potenzial der Selektivvertragsoptionen mindern. Dazu gehören ungenügend gelöste Bereinigungsprobleme der hausarztzentrierten Versorgung, besonderen ambulanten Versorgung und - jenseits und nach der Ausschöpfung 1\%-Anschubfinanzierung - auch der integrierten Versorgung. Auch die Potenziale der Rabattregelungen im Arzneimittelsektor werden begrenzt bleiben, da den Kassen nach wie vor keine Mengensteuerung möglich ist. Es finden sich jedoch u.a. auch Maßnahmen, die für die Vertragsbeziehungen zwischen Kassen und Leistungsanbieter in eine konkret antiwettbewerbliche Reformrichtung weisen. Dazu gehören die Neuregelungen der ambulanten Krankenhausbehandlung (\$ 116b) und der künftigen vertragsärztlichen Vergütung. In die gleiche Richtung gehen auch planerische Detailvorgaben für die Verwendung der Anschubfinanzierung der integrierten Versorgung sowie die Angebotspflicht und Gestaltungsvorgaben für die hausarztzentrierte Versorgung. Wirklich umfangreiche wettbewerbliche Handlungsspielräume sind im Hilfsmittelbereich erwartbar, denn hier soll eine konsequente Umstellung auf ein vertragswettbewerbliches Modell erfolgen.

Dass diesem Reformvorhaben kein konsistenter Wettbewerbsansatz zu Grunde liegt, zeigt sich auch bei einem Blick auf den Spitzenverband Bund der Krankenkassen. So soll der neue Verband als Kollektivvertragspartner „alle nicht wettbewerblichen Verträge mit Leistungserbringern“ (Begründung Gesetzentwurf BT 16/3100, Allgemeiner Teil, Punkt II.9.) schließen. Die im Zuge dessen vorgesehenen Einflussmöglichkeiten des Verbands werden zu stärkeren Vereinheitlichungen von Versorgung und Vergütung führen. Damit steht der Spitzenverband dann nicht nur im Konflikt mit dem allgemeinen Reformziel der „Wettbewerbstärkung“, sondern durchaus auch mit einem eigenen Aufgabenfeld, wonach er „die Rahmenbedingungen für einen intensiveren Wettbewerb" (Konsenspapier: Zentrale Inhalte der Gesundheitsreform 2006, S. 6) regeln soll. Inwieweit aus diesen beiden Rollen auch noch Konflikte mit seiner Rolle als Interessenvertreter der Kassen auf Bundesebene entstehen, ist noch nicht abzusehen. Widersprüche zwischen den verschiedenen Rollen des neuen Spitzenverbands sind aber keinesfalls auszuschließen.

Unter dem Strich lässt sich für die geplante Gesundheitsreform und den Wettbewerb festhalten, dass der Gesetzentwurf viel wettbewerbliche Rhetorik und wenig wettbewerbliche Substanz bietet. Die Zukunft ist daher absehbar. Der Bedarf an finanzierungsseitigen und leistungsseitigen Strukturreformen wird auch nach dieser Reform fortbestehen. Weitere Gesundheitsreformen werden wahrscheinlich alsbald wieder anstehen.

\section{Literatur}

Cassel, D. (1993): Anspruch und Wirklichkeit. Zur Organisationsreform der GKV. In: Soziale Sicherheit, Heft 1/1993, S. 18-24.

Cassel, D./Ebsen, I./Greß, S./Jacobs, K./Schulze, S./Wasem, J. (2006) Weiterentwicklung des Vertragswettbewerbs in der GKV - Vorschläge für kurzfristig umsetzbare Reformschritte, Gutachten im Auftrag des AOK-BV, Juli 2006.

CDU/CSU/SPD: Gemeinsam für Deutschland - mit Mut und Menschlichkeit. Koalitionsvertrag zwischen CDU, CSU und SPD, vom 11. November 2005

Ebsen, I./Greß, S./Jacobs, K./Szecsenyi, J./Wasem, J. (2003): Vertragswettbewerb in der gesetzlichen Krankenversicherung zur Verbesserung von Qualität und Wirtschaftlichkeit der Gesundheitsversorgung, AOK im Dialog Bd. 13, Bonn, S. 145-307.

Eckpunkte zu einer Gesundheitsreform 2006 vom 04. Juli 2006

Gesetzentwurf der Fraktionen der CDU/CSU und SPD: Entwurf eines Gesetzes zur Stärkung des Wettbewerbs in der Gesetzlichen Krankenversicherung (GKV-Wettbewerbsstärkungsgesetz - GKVWSG), Bundestagsdrucksache 16/3100, 24. Oktober 2006.

IGES (Institut für Gesundheits- und Sozialforschung $\mathrm{GmbH}$ )/ Cassel, D./Wille, E./WIdO (wissenschaftliches Institut der AOK) (2006): Steuerung der Arzneimittelausgaben du Stärkung des Forschungsstandortes für die pharmazeutische Industrie. Gutachten für das Bundesministerium für Gesundheit, 2. Juni 2006.

IGES (Institut für Gesundheits- und Sozialforschung $\mathrm{GmbH}$ )/Lauterbach, K. W./ Wasem, J. (2004): Klassifikationsmodelle für Versicherte im Risikostrukturausgleich. Untersuchung zur Auswahl geeigneter Gruppenbildungen, Gewichtungsfaktoren und Klassifikationsmerkmale für einen direkt morbiditätsorientierten Risikostrukturausgleich 
i der gesetzlichen Krankenversicherung, im Auftrag des Bundesministeriums für Gesundheit und Soziale Sicherung, Endbericht, November 2004.

Jacobs, K./Reschke, P./Cassel, D./Wasem, J. (2002): Zur Wirkung des Risikostrukturausgleichs in der gesetzlichen Krankenversicherung. Eine Untersuchung Auftrag des Bundesministeriums für Gesundheit, Band 140 Schriftenreihe des Bundesministeriums für Gesundheit, Nomos Verlagsgesellschaft 2002.

Konsenspapier „Zentrale Inhalte der Gesundheitsreform 2006" vom Oktober 2006.

\section{Fußnoten}

1 Dieser Beitrag basiert auf dem Gesetzentwurf der Fraktionen der CDU/CSU und SPD vom 24.10.2006, Bundestagsdrucksache 16/3100.

2 Vgl. z.B. Jacobs et al. 2002.
3 Vgl. z.B. Cassel et al. 2006 ; Ebsen et al. 2003.

4 Siehe Begründung zum Gesetzentwurf BT 16/3100, zu Nummer 161 (§ 242).

5 Vgl. z.B. bereits Cassel 1993.

6 Vgl. zu einer umfassenden Übersicht über die bisherigen Selektivvertragsoptionen, ihre Umsetzung und Problemanalyse Cassel et al. 2006.

7 Vgl. zu einem solchen Konzept Cassel et al. 2006; Ebsen et al. 2003.

8 Zwar können Krankenhäuser in der integrierten Versorgung eine ambulante Versorgung der Leistungen nach $\S 116 \mathrm{~b}$ auch im Rahmen von selektiven Verträgen erbringen, allerdings unterliegen die Kassen grundsätzlich den Kollektivverpflichtungen des neuen $§ 116 \mathrm{~b}$.

9 Und damit deutlich länger als die mit dem Vertragsarztrechtsänderungsgesetz beschlossene Verlängerung der 1\%- Anschubfinanzierung um zwei Jahre.

10 vgl. IGES/WIdO/Cassel/Wille 2006.

\section{Das neue Standardwerk}

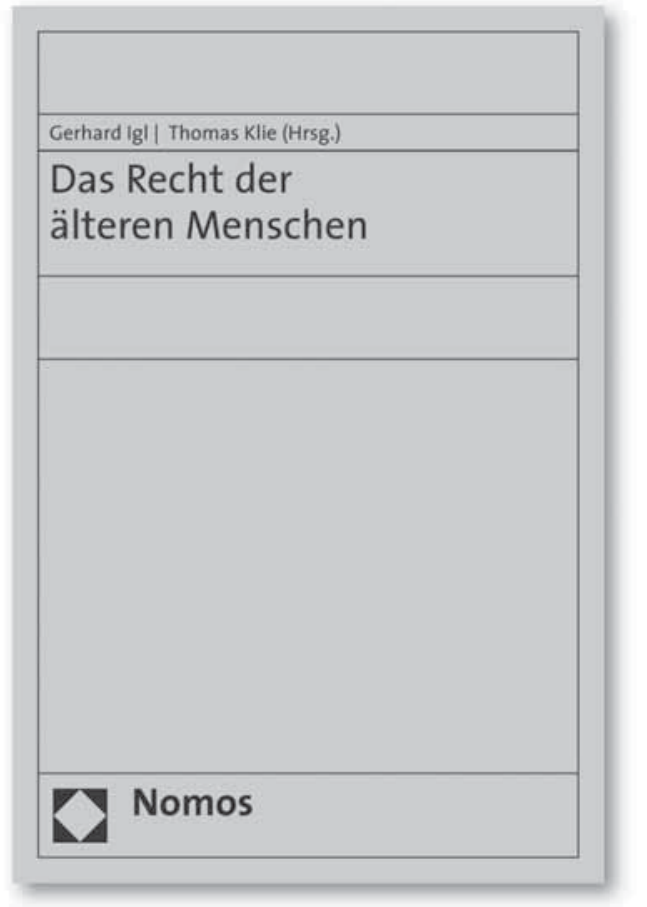

\section{Das Recht der älteren Menschen}

Herausgegeben von Prof. Dr. Gerhard Igl, Universität Kiel und Prof. Dr. Thomas Klie, Ev. Fachhochschule Freiburg

2007, ca. 265 S., brosch., ca. 34, -€

ISBN 978-3-8329-2239-9

Erscheint Januar 2007

Die Zahl älterer Menschen wächst in Deutschland und überall in Europa. Das vorliegende Buch macht deutlich, wie sich der demografische Wandel auf das Recht auswirkt. Namhafte juristische Autoren zeigen die vielfältigen Fragestellungen auf, die ältere Menschen betreffen und die Rechtswissenschaft herausfordern. Das Werk richtet sich vor allem an Beratungsinstitutionen im Sozial- und Gesundheitswesen, Verbraucherschutzverbände sowie Einrichtungen und Dienste für ältere Menschen.

\section{Die Autoren:}

Axel Bauer | Prof. Dr. Gerhard Nägele, Universität Dortmund | Prof. Dr. Peter Udsching, BSG Kassel | Prof. Dr. Hans F. Zacher, MaxPlanck-Institut für ausländisches und internationales Sozialrecht, München | Prof. Dr. Gisela Zenz, Universität Frankfurt/M.

Bitte bestellen Sie bei Ihrer Buchhandlung oder bei: Nomos Verlagsgesellschaft 76520 Baden-Baden | www.nomos.de 